\title{
Case Report: Refractory Cryptosporidiosis after CAR T-Cell Therapy for Lymphoma
}

\author{
Caitlin A. Trottier, ${ }^{1}$ Christina F. Yen, ${ }^{2}$ Grace Malvar, ${ }^{3}$ Jon Arnason, ${ }^{4}$ David E. Avigan, ${ }^{5}$ and Carolyn D. Alonso ${ }^{2 *}$ \\ ${ }^{1}$ Department of Medicine, Beth Israel Deaconess Medical Center, Harvard Medical School, Boston, Massachusetts; ${ }^{2}$ Division of Infectious \\ Diseases, Beth Israel Deaconess Medical Center, Harvard Medical School, Boston, Massachusetts; ${ }^{3}$ Division of Pathology, Beth Israel \\ Deaconess Medical Center, Harvard Medical School, Boston, Massachusetts; ${ }^{4}$ Department of Medical Oncology, Beth Israel Deaconess Medical \\ Center, Harvard Medical School, Boston, Massachusetts; ${ }^{5}$ Division of Hematology and Hematologic Malignancies, Beth Israel Deaconess Medical \\ Center, Harvard Medical School, Boston, Massachusetts
}

\begin{abstract}
Cryptosporidial diarrhea is uncommon in immunocompetent individuals, more often seen in severely immunocompromised patients. Severe refractory cases have been described in patients with HIV/AIDS before the advent of modern antiretroviral therapy due to an inability to mount an adequate cellular immune response. We describe an 85year-old patient post-chimeric antigen receptor T-cell therapy relapsed lymphoma who developed refractory Cryptosporidium spp. diarrhea in the setting of persistent CD4+ cytopenia. Despite receiving multiple antiparasitic agents, including failure of a prolonged course of nitazoxanide, the patient experienced persistent symptoms for 9 months with repeatedly positivity stool Cryptosporidium spp. direct fluorescent antibody (DFA) test. We highlight this case of refractory Cryptosporidium spp. and the importance of recognizing the pathogen in a non-HIV-infected immunosuppressed host.
\end{abstract}

An 85-year-old woman with a history of relapsed transformed diffuse large B-cell lymphoma post-chimeric antigen receptor $\mathrm{T}(\mathrm{CAR} \mathrm{T})$-cell therapy 2 years earlier presented to the hospital for initiation of intravenous antiviral therapy for cytomegalovirus (CMV) retinitis after recent treatment with valganciclovir. The patient had an extensive treatment course for her lymphoma that included ibritumomab tiuxetan, nivolumab, lenalidomide, idelalisib, obinutuzumab, and multiple courses of rituximab and bendamustine over 9 years. When her disease transformed to an aggressive histology and failed to respond to two cycles of dose adjusted $\mathrm{EPOCH}$ with rituximab, she proceeded with salvage lisocabtagene maraleucel, a CD-19-specific CAR T cell. As part of undergoing CAR T-cell therapy, the patient underwent leukopheresis to harvest CD4 and CD8 cells; however, CD4 CAR T-cells could not be manufactured, and the patient received only CD8 CAR T cells with complete remission of her disease.

Her post-CAR T-course was complicated by low CD4 counts (ranging between 40 and $250 / \mu \mathrm{L}$ ), hypogammaglobulinemia (immunoglobulin G level range 300-500 mg/dL) for which she received monthly immunoglobulin replacement, and an episode of Pneumocystis jiroveci pneumonia. At the time of admission, she reported having several days of diarrhea that had started with valganciclovir initiation.

Initial concerns were for CMV colitis or valganciclovirassociated diarrhea. However, to further evaluate her symptoms, additional stool studies were sent including testing for Salmonella, Shigella, Campylobacter, Escherichia coli O157:H7, Vibrio, Yersinia, Microsporidia, C. difficile, and ova and parasites; all were negative. However, fecal direct fluorescent antigen (DFA) for Cryptosporidium/Giardia was positive for the presence of Cryptosporidium spp. oocysts. Treatment with nitazoxanide 500 mg twice daily was initiated. The patient continued to experience ongoing largevolume diarrhea (upwards of $2 \mathrm{~L}$ of stool output a day) despite 14 days of nitazoxanide. A flexible sigmoidoscopy

\footnotetext{
*Address correspondence to Carolyn D. Alonso, Beth Israel Deaconess Medical Center, Harvard Medical School, 110 Francis Street, Suite GB, Boston, MA 02215. E-mail: calonso@bidmc. harvard.edu
}

was performed to evaluate for other causes of diarrhea. This demonstrated scattered diverticuli but no colitis. Biopsies were not obtained at the time.

The patient improved and was briefly off of therapy until she reported a return of diarrheal stools (Figure 1). Repeat stool testing demonstrated the persistence of Cryptosporidium spp. oocysts. Nitazoxanide $500 \mathrm{mg}$ twice daily and azithromycin $500 \mathrm{mg}$ daily was initiated. Despite 4 weeks of combined therapy, symptoms persisted, and follow-up DFA for Cryptosporidium spp. remained positive. She was trialed on paromomycin $500 \mathrm{mg}$ four times daily. Two months after the initial diagnosis, she underwent esophagogastroduodenoscopy (EGD) with colonoscopy. EGD demonstrated erosions in the stomach and duodenal bulb. Colonoscopy showed patchy erythema in the terminal ileum, ileocecal valve, and rectum. Tissue pathology confirmed active enteritis with organisms consistent with Cryptosporidium spp. throughout the terminal ileum, ileocecal valve, colon, and rectum (Figure $2 \mathrm{~A}$ and $\mathrm{B})$. Immunostaining for CMV was negative.

As such, the cause of her diarrhea was attributed to refractory cryptosporidial infection. Bovine colostrum (DiaResQ 4 g-35 kcal/7 g oral powder packet three times daily) was added to the regimen of paromomycin and nitazoxanide. However, the patient continued to have large-volume diarrhea. An Investigational New Drug Application request to the U.S. Food and Drug Administration was obtained to initiate clofazimine (100 mg every 8 hours for 5 days, followed by maintenance dose of $100 \mathrm{mg}$ daily) based on early in vitro data suggesting activity against the pathogen. ${ }^{8}$ After several months on clofazimine, paromomycin, and bovine colostrum, her diarrhea persisted; at that point, azithromycin and nitazoxanide were restarted, along with rifaximin $(550 \mathrm{mg}$ three times daily). After about a month of combined treatment with paromomycin, nitazoxanide, clofazamine, azithromycin, rifaximin, and bovine colostrum, she continued to have diarrhea and was considered to represent treatment failure. All antiparasitic agents were discontinued (Figure 1). She was started on total parenteral nutrition and antimotility therapy was maximized. She had minimal symptomatic improvement but was able to be safely discharged home after a 3-month hospitalization. However, due to protein and 


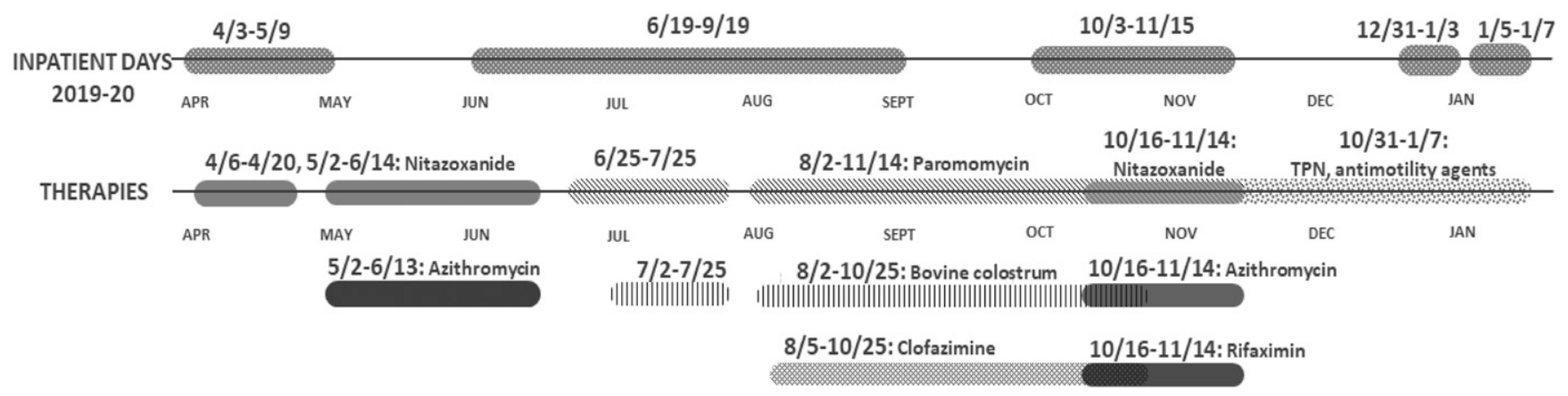

FIGURE 1. Hospitalization and treatment course from April 2019 through January 2020.

calorie malnutrition secondary to her parasitic infection, she developed hypoalbuminemia, resulting in edema, a large right-sided pleural effusion, and shortness of breath. She was readmitted to the hospital and ultimately died after developing rapidly progressive respiratory failure. Post-mortem flow cytometry from the pleural effusion demonstrated evidence of relapsed lymphoma, which was ultimately considered to be the primary cause of death.

\section{DISCUSSION}

Cryptosporidium spp. are obligate intracellular protozoa often causing gastrointestinal disease; with Cryptosporidium hominis and parvum more frequently observed in developing countries compared with the prevalence in United States. However, the impact is felt globally, especially in children and immunocompromised patients. ${ }^{1}$ Severe or refractory cryptosporidiosis despite antiparasitic therapy was previously seen in patients with HIV before the advent of modern antiretroviral therapy due to an inability to recover the cellular immune response, which prevents repeated infection. ${ }^{2-4}$

CAR T is a personalized cellular immunotherapy for cancer in which a patient's $T$ cells are genetically modified through the insertion of a chimeric T-cell receptor comprising a single chain variable region targeting a tumor associated protein and costimulatory and activation signals that promote T-cell activation and expansion. Lisocabtagene maraleucel targets the CD19 surface protein and has demonstrated clinical efficacy in aggressive B cell lymphomas, resulting in dramatic tumor regression and durable remission in a subset of patients with relapsed/refractory disease. ${ }^{5}$ Treatmentassociated toxicities include cytokine release syndrome and neurotoxicity related to the dramatic expansion of activated T cells and associated release of inflammatory cytokines. ${ }^{6,7}$ Prolonged myelosuppression and hypogammaglobulinemia have been noted related to preceding lymphodepleting chemotherapy, cytokine-mediated suppression of bone marrow hematopoiesis after CAR T-cell expansion, and targeted eradication of $B$ cells due to the direct effect of the CAR T cells on the healthy B-cell compartment. As a result, opportunistic fungal and viral infections, and viral-mediated processes such as PM progressive multifocal leukoencephalopathy have been observed with CAR T-cell therapy. ${ }^{8-10}$

The development of cryptosporidiosis in patients receiving CAR $T$ has not been documented, making this the first reported case. In the case of our patient, years of lymphocyte-depleting chemotherapy, notably bendamustine after age 65, resulted in diminished bone marrow and lymphocyte recovery. ${ }^{11}$ This effect may have been exacerbated by the CAR T therapy, increasing her risk for cryptosporidiosis. ${ }^{12}$ Of note, during the cell harvesting phase of her CAR T in 2017, she had adequate CD8 cells collected, but she had

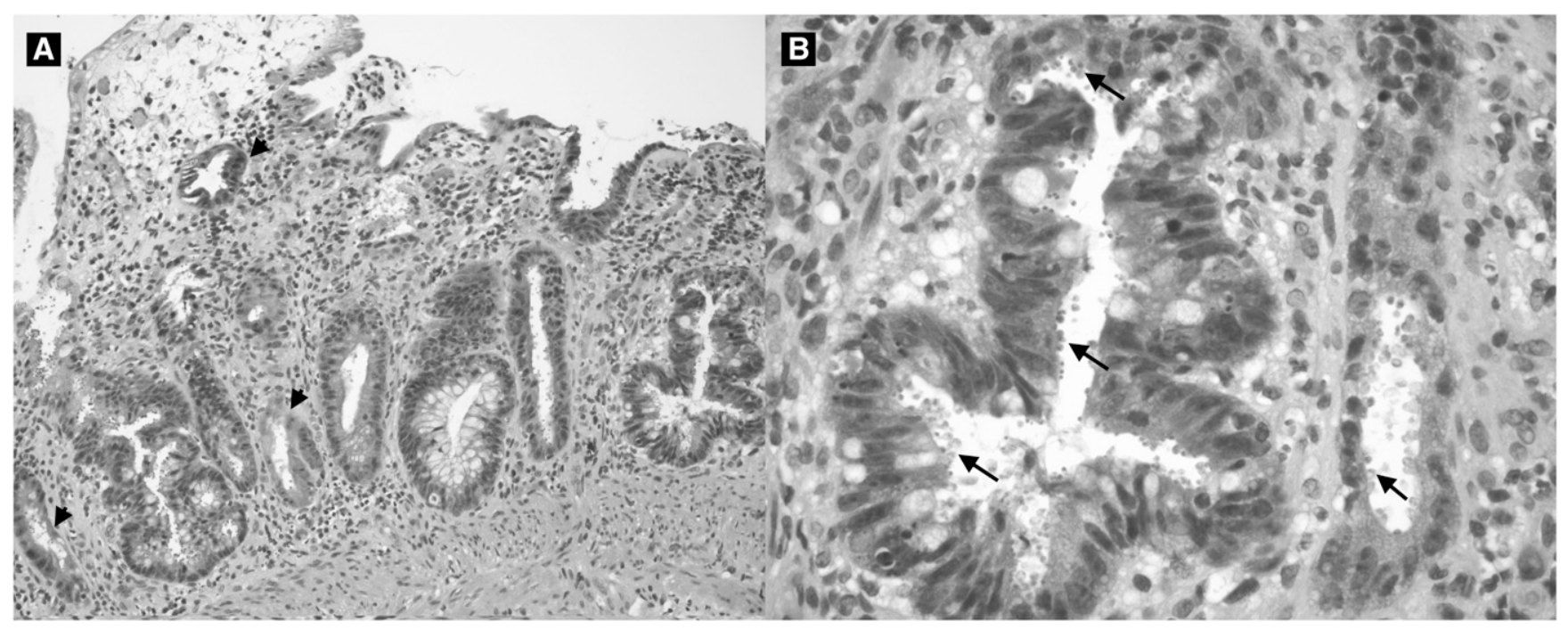

FIGURE 2. (A) Colonic mucosa with active colitis, crypt atrophy and dropout. (B) Cryptosporidium spp. oocysts lining the crypt epithelium. This figure appears in color at www.ajtmh.org. 
few to no CD4 cells collected. This finding, in combination with the high rates and durability of responses to CAR T cells in indolent lymphomas, highlights the need to consider CAR T therapy early to avoid the additive immunological insult of multiple lines of therapy. ${ }^{13}$ Novel immunotherapies such as CAR T therapy are becoming more commonplace, and it is necessary to increase provider awareness of cryptosporidiosis as a potential cause of acute or chronic diarrhea as a result of long-term, immunotherapy-related perturbations to immune activity.

Adequate curative therapy for cryptosporidial diarrhea remains elusive and represents a serious unmet medical need. We postulate that the diminished efficacy of antiparasitic agents in the HIV/AIDS patient population may be extrapolated to patients with severe immune suppression, especially those with depressed CD4 counts, as in this case. The data for effective treatment of severe cryptosporidiosis in patients living with HIV/AIDS without immune reconstitution is limited. Reconstitution of the immune system with antiretroviral therapy has been a mainstay in the treatment of cryptosporidiosis in HIV-infected individuals; however, in cases such as the one presented here, immune reconstitution may not be possible due to underlying marrow suppression. Nitazoxanide with or without azithromycin is usually the first-line treatment, given findings that suggest the addition of azithromycin has effectiveness in HIV- negative patients. ${ }^{14}$ Rifaximin has been shown to be effective in an HIV patient population in a very small study. ${ }^{15}$ This study excluded patients with CD4 counts $\leq 200 / \mu \mathrm{L}$, limiting its generalizability to patients with more severe immune suppression. Paromomycin has been reported to have some efficacy against Cryptosporidium spp., although it was found to be inferior compared with nitazoxanide. ${ }^{14}$ Historically used to treat leprosy and multidrug-resistant tuberculosis, clofazime was explored as a novel treatment agent. Given recent in vitro and mouse model studies suggesting efficacy against Cryptosporidium spp., a clinical trial in Malawi trialed clofazamine as a treatment of cryptosporidial diarrhea in HIV-infected patients. ${ }^{14,16}$ Unfortunately, the trial was not completed due to an inability to enroll enough patients. ${ }^{16}$ Althogh clofazimine did not prove to be efficacious in our patient, it is important to continue exploring other avenues of treatment given the limited number of options available. For instance, in early 2021, data were published demonstrating that combination therapy with secnidazole-nitazoxanide was effective in reducing oocyst shedding and intestinal histopathology in both immunocompromised and immunocompetent mouse models. ${ }^{17}$ Although these data were published 2 years after our case, it is reassuring to note that new therapeutic options are continuing to be explored to expand our future treatment options.

Although an uncommon occurrence, severe cryptosporidiosis is an infection with significant consequences in immunocompromised hosts. Further resources for drug development and clinical trials are necessary to address this organism and manage patients such as ours who did not respond to standard therapies.

Received February 27, 2021. Accepted for publication March 22, 2021.

Published online July 8, 2021.
Authors' addresses: Caitlin Trottier, Internal Medicine, Beth Israel Deaconess Medical Center, Boston, MA, E-mail: catrotti@bidmc. harvard.edu. Christina F. Yen, Infection Control, Beth Israel Deaconess Medical Center, Boston, MA, E-mail: cyen@bidmc. harvard.edu. Grace Malvar, Pathology, Beth Israel Deaconess Medical Center, Boston, MA, E-mail: gmalvar@bidmc.harvard.edu. David E. Avigan, Hematology and Hematologic Malignancies, Beth Israel Deaconess Medical Center, Boston, MA, E-mail: davigan@bidmc.harvard.edu. Jon Arnason, Beth Israel Deaconess Medical Center, Oncology, Boston, MA, E-mail: jarnason@bidmc. harvard.edu. Carolyn D. Alonso, Beth Israel Deaconess Medical Center, Infectious Diseases, Boston, MA, E-mail: calonso@bidmc. harvard.edu.

\section{REFERENCES}

1. Current WL, Garcia LS, 1991. Cryptosporidiosis. Clin Microbiol Rev 4: 325

2. Pantenberg B et al., 2008. Intestinal immune response to human Cryptosporidium sp. Infection Infect Immun 76: 23-29.

3. Brudno JN, Kochenderfer JN, 2016. Toxicities of chimeric antigen receptor $\mathrm{T}$ cells: recognition and management. Blood 127: $3321-3330$.

4. Brudno JN et al., 2020. Safety and feasibility of anti-CD19 CAR $\mathrm{T}$ cells with fully human binding domains in patients with B-cell lymphoma. Nat Med 26: 270-280 (Erratum in: Nat Med. 2020 May;26(5):803, PMID: 31959992).

5. Abramson JS et al., 2020. Lisocabtagene maraleucel for patients with relapsed or refractory large B-cell lymphomas (TRANSCEND NHL 001): a multicentre seamless design study. Lancet 396: 839-852.

6. Neelapu SS et al., 2018. Chimeric antigen receptor T-cell therapy-assessment and management of toxicities. Nat Rev Clin Oncol 15: 47-62 (Epub 2017 Sep 19).

7. Neelapu SS et al., 2017. Axicabtagene ciloleucel CAR T-cell therapy in refractory large b-cell lymphoma. $N$ Engl $J$ Med 377: 2531-2544.

8. Wudhikarn $\mathrm{K}$ et al., 2020. Infection during the first year in patients treated with CD19 CAR T cells for diffuse large B cell lymphoma. Blood Cancer J 10: 79.

9. Hill JA et al., 2018. Infectious complications of CD19-targeted chimeric antigen receptor- modified T-cell immunotherapy. Blood 131: 121-130.

10. Bhoj VG, Arhontoulis D, Wertheim G, 2016. Persistence of long-lived plasma cells and humoral immunity in individuals responding to CD19-directed CAR T-cell therapy. Blood 128: 360-370.

11. Fung $M$ et al., 2019. Increased risk of infectious complications in older patients with indolent non-hodgkin lymphoma exposed to bendamustine. Clin Infect Dis 68: 247-255.

12. Saito H, Maruyama D, Maeshima AM, 2015. Prolonged lymphocytopenia after bendamustine therapy in patients with relapsed or refractory indolent B-cell and mantle cell lymphoma. Blood Cancer J5: e362.

13. Hirayama AV et al., 2019. High rate of durable complete remission in follicular lymphoma after CD19 CAR-T cell immunotherapy. Blood 134: 636-640.

14. Chavez MA, White AC Jr., 2018. Novel treatment strategies and drugs in development for cryptosporidiosis. Expert Rev Anti Infect Ther16: 655-661.

15. Amenta $M$ et al., 1999. Intestinal protozoa in HIV-infected patients: effect of rifaximin in Cryptosporidum parvum and Blastocystis hominis infections. J Chemother 11: 391-395.

16. Nachipo $P$ et al., 2018. Evaluating the safety, tolerability, pharmacokinetics and efficacy of clofazimine in cryptosporidiosis (CRYPTOFAZ): study protocol for a randomized controlled trial. Trials 19: 456.

17. Madbouly N, El Amir A, Abdel Kader A, Rabee I, Farid A, 2021. The immunomodulatory activity of secnidazole-nitazoxanide in a murine cryptosporidiosis model. J Med Microbiol 70 (Epub 2021 Feb 23). doi: 10.1099/jmm.0.001327. 